\title{
REVISIONES
}

\section{MED: Modelo de formación continua para profesores de matemática, basada en la experiencia*}

\author{
MED: in-service instructional model for mathematics teachers based on experience
}

\author{
Horacio Solar $^{a}$, Andrés Ortiz ${ }^{b}$, Rodrigo Ulloa ${ }^{c}$ \\ a Pontificia Universidad Católica de Chile. Correo electrónico: hsolar@uc.cl \\ b Universidad Católica de la Santísima Concepción. Correo electrónico: rulloa@ucsc.cl \\ c Universidad Católica de la Santísima Concepción. Correo electrónico: aortiz@ucsc.cl
}

\section{RESUMEN}

Este artículo presenta un modelo de formación continua para el profesor que enseña matemática, que hemos llamado "Mejoramiento de la Experiencia Docente" MED, el cual ha demostrado ser exitoso para producir apropiación de un Conocimiento Pedagógico del Contenido. De las diversas experiencias de formación continua a profesores que hemos desarrollado, nos focalizaremos en una sobre gestión de la argumentación en el aula de matemáticas para describir las etapas, principios y características del modelo MED, destacando el uso de una progresión articulada de estudio de casos, donde la elicitación de ideas de los profesores en contextos donde la práctica es el foco central para analizar y modificar la experiencia docente, lo cual permite superar los elementos de un proceso de formación continua tradicional. El modelo MED es transferible para que pueda ser utilizado por otros formadores.

Palabras clave: formación continua, conocimiento pedagógico del contenido, conocimiento del profesor, argumentación en el aula de matemáticas.

\section{ABSTRACT}

This paper presents an in-service instructional model for mathematics teachers, called "Improvement of the Teaching Experience", MED, which has resulted successful in promoting the development of pedagogical content knowledge. From the various experiences of in-service teachers' education which we have developed, the focus will be on those related to the orchestration of argumentation in the classroom allowing a description of the stages, principles and characteristics of the model, remarking the use of an articulated progression of case studies, where eliciting beliefs and ideas about teaching practices results as a central task to analyze and modify the teaching experience, surpassing the elements of a traditional model. The MED model can be transferred to be used by others teachers educators.

Key Words: in-service teacher education, pedagogical content knowledge, teacher knowledge, argumentation in the mathematics classroom 


\section{ANTECEDENTES SOBRE LA FORMACIÓN CONTINUA DEL PROFESOR}

Pese a la importancia que se le ha dado a la formación continua de profesores que enseñan matemáticas, su impacto no se ha reflejado en los desempeños de profesores y tampoco en los estudiantes de enseñanza media y básica. Es importante precisar que la formación continua de profesores en Chile es realizada principalmente por instituciones no gubernamentales que dan Asistencia Técnica Educativa (ATE) a establecimientos educacionales, las que actualmente suman más de 900, con diferentes esquemas de propiedad. Estas son instituciones con o sin fines de lucro, pertenecientes a privados, fundaciones y universidades.

Los bajos impactos en la formación continua se pueden observar en los resultados del estudio TEDS-M. En este participaron profesores chilenos que hacen docencia en matemática, revelando un muy bajo nivel de conocimientos para la enseñanza (Ávalos y Matus, 2010). Por otra parte, los informes de resultados de los estudiantes en las pruebas estandarizadas internacionales PISA (OCDE, 2013) muestran que los desempeños en matemáticas a nivel nacional y sudamericano están bajo el promedio de la OCDE y las pruebas estandarizadas nacionales (Agencia de la Calidad de la Educación, 2013) señalan que hay un estancamiento en el desarrollo matemático de los estudiantes.

Al evaluar el proceso de formación de los profesores en ejercicio en nuestro país, la OCDE ha señalado la necesidad de incorporar métodos más progresistas de enseñanza por parte de las instituciones formadoras de profesores, además de una mayor cercanía con la problemática de la enseñanza en el medio escolar (Ministerio de Educación, 2005). Subyace, además, "la creencia de la mayoría de los académicos que para enseñar lo único que se requiere es conocer el contenido o materia" (Ministerio de Educación, 2005: 46). Si bien estos antecedentes son de hace 10 años, investigaciones recientes han reportado resultados que no sólo han complementado y corroborado el diagnóstico anterior, sino que han detectado que varias de estas deficiencias se han mantenido hasta hoy en formación continua (Bellei, Contreras y Valenzuela, 2008; Donoso, 2008; Bellei, Osses y Valenzuela, 2010; Bellei, Raczynski y Valenzuela, 2010; Alvarado, Cabezas, Falck y Ortega, 2012; Valenzuela, Villarroel y Villalobos, 2013). Estos antecedentes muestran que los programas de formación continua en Chile no están modificando las prácticas de los profesores, ni manifiestan cambios en los resultados de aprendizaje de sus estudiantes. Esta realidad no es distinta a nivel internacional, en que gran parte de las experiencias de formación continua de profesores de matemáticas no ha impactado significativamente en los docentes porque estos no han cambiado sus prácticas en relación a las innovaciones curriculares (Roesken, 2011). A ello se suma que los desempeños de los alumnos tampoco han variado significativamente en la última década.

Consideramos que el poco impacto en los procesos de formación continua de profesores que enseñan matemáticas se relaciona con procesos que no cuentan con un modelo de formación declarado, explícito y consciente, y que además tienen características tradicionales de lo que sería un proceso de enseñanza-aprendizaje en el aula, es decir una relación vertical entre formador y profesor en vez de promover una relación de colaboración. Lo anterior se ejemplifica en que el formador transmite al profesor qué y cómo enseñar en vez de promover otros mecanismos tales como prácticas consistentes con el modelo declarado; enseña estrategias de enseñanza sin conectar con las experiencias de que ha tenido el profesor en su práctica. Esta visión del proceso instruccional puede emerger cuando no se cuenta con un modelo de formación continua. 
Si bien a nivel internacional existe un creciente interés por la formación continua de profesores, la literatura se centra en experiencias exitosas de formación más que modelos de formación continua. Un ejemplo de ello se ve reflejado en las actas de ICME 12 (Cho, 2015) realizado en Tokio, en donde el grupo destinado a la formación continua era el que contaba con más trabajos a discutir de las sesiones regulares del grupo, los que mostraban una variedad de experiencias realizadas sobre formación continua. Entre ellas destacaban el uso de estudio de clases, el uso de videos para promover la reflexión en el profesor y la importancia de trabajar con la experiencia de los profesores. No obstante, eran escasos los trabajos que presentaban un modelo de desarrollo profesional en que se analizara las características de dicho modelo.

El grupo de investigación "Competencias Matemáticas" (COMMAT) ha venido realizando investigaciones en el contexto del desarrollo profesional del profesor que enseña matemáticas, particularmente para el desarrollo de competencias matemáticas en estudiantes de enseñanza básica. Entre ellas, nos ha interesado indagar en la formación en prácticas docentes que promueven el desarrollo de la argumentación en el aula matemática (Solar y Deulofeu, en prensa). En estas experiencias, COMMAT ha ido desarrollando una metodología de trabajo con los docentes caracterizada por problematizar situaciones de enseñanza por medio del análisis de prácticas de aula, incentivando la reflexión permanente de los profesores participantes, teniendo como objetivo que profundicen en las reflexiones respecto a las prácticas, tanto de sus colegas como las propias, en un contexto de trabajo colaborativo, en que el formador va colaborando con el docente en la preparación de sus clases y va retroalimentado el proceso mediante la observación de sus clases. Dicha metodología se ha llevado a cabo por medio de un ciclo de formación continua con profesores que, más que avanzar linealmente por fases, supone un trabajo en espiral, en que la ejecución de cada fase permite profundizar en las fases anteriores. Los resultados obtenidos nos han llevado a la formulación del modelo de desarrollo profesional llamado "Mejoramiento de la Experiencia Docente" (MED) cuya creación se ha hecho sobre la base de preguntarnos cuáles son las características fundamentales de este modelo de desarrollo profesional que vaya más allá del contexto de formación para la promoción de competencias matemáticas, teniendo como consecuencia que puede ser utilizado para promover desarrollo en los profesores en su Conocimiento Pedagógico del Contenido (CPC) en el aula de matemáticas.

En este artículo, analizaremos los principios, etapas y características del MED por medio de una de las experiencias de desarrollo profesional centrado en prácticas docentes para promover la argumentación en el aula de matemáticas.

\section{DESARROLLO PROFESIONAL DEL PROFESOR DE MATEMÁTICA}

Hemos mencionado resultados empíricos que muestran que en Chile los profesores muestran grandes dificultades para transferir al aula matemática los conocimientos adquiridos en los procesos de capacitación, ya que ello supone generar cambios paradigmáticos en su ejercicio docente. De los procesos de formación que han obtenido resultados positivos a nivel internacional, Roesken (2011) señala que el profesor aprende estableciendo relaciones entre la teoría y la práctica, en donde su conocimiento profesional se desarrolla contrastando creencias y experiencias en contextos reflexivos y colaborativos. En consecuencia, se requiere poner un especial foco en los modelos que indagan y abordan las prácticas de enseñanza. 
Roesken (2011) destaca 4 modelos de formación continua, construidos todos a partir de experiencia empírica y teorización: (1) Schoenfeld (2011) propone un modelo de formación para profesores de matemáticas estructurado en tres focos: recursos del profesor (por ejemplo, sus conocimientos), propósitos u objetivos que tiene un docente, y orientaciones (concepciones, toma de decisiones). Schoenfeld relata cómo ha desarrollado procesos de formación en profesores por medio de estos tres focos, por ejemplo, en el estudio de la indagación en la clase de matemáticas; (2) el modelo de aspectos profesionales (Llinares y Kramer, 2006), que sitúa al profesor en formación como un actor social, identificando tres materias de estudio: contenido, contexto y comunidades; (3) Krainer (2002) propone un modelo de cuatro dimensiones de desarrollo profesional: acción, reflexión, autonomía y participación en redes de colaboración; (4) Finalmente, Clarke y Hollingsworth (2002) proponen avanzar desde enfoques tradicionales de formación, que primero pretenden cambiar los conocimientos y concepciones de los profesores, para luego cambiar las prácticas de clases, hacia modelos interconectados de desarrollo profesional, que involucran activamente al profesor en la aplicación y reflexión sobre dominios de conocimiento personales, externos, de la práctica y de resultados. En esta misma línea, Guskey (2000) sostiene un camino inverso al enfoque tradicional de formación docente, en que primero se debe modificar las prácticas de los docentes, recogiendo evidencia de los cambios de sus estudiantes y, en consecuencia, produciéndose cambios en las actitudes y concepciones del docente.

Se observa además que todos estos modelos incluyen al conocimiento como un elemento central de la formación. La calidad y características de aquel conocimiento propio de un profesor de matemáticas ha sido motivo de amplio debate e investigación en la comunidad académica, en donde destacan los ampliamente conocidos aportes de los modelos del Conocimiento Pedagógico del Contenido [CPC] (Shulman, 1986) y el Conocimiento Matemático para la Enseñanza (Hill, Ball y Schilling, 2008), en adelante utilizaremos el término acuñado por Shulman "conocimiento pedagógico del contenido" como concepto aglutinador de los conocimientos que pone en juego el profesor en el aula en un proceso de enseñanza aprendizaje, entendiendo que este concepto, incorpora varios tipos de conocimientos: enseñanza de las matemáticas, comprensión de los estudiantes sobre la matemática, currículo de matemáticas, la evaluación de las matemáticas y estrategias para la gestión del aula de matemáticas (Vergara y Cofre, 2014).

Diversas iniciativas de desarrollo profesional proyectan características de una formación continua en matemática. Lave y Wenger (1991) identifican varias propuestas de formación de profesores basan su metodología de enseñanza en la perspectiva de cognición situada, en que existe un cierto consenso en que el desarrollo profesional debe situarse en la práctica (An y Peter-Koop, 2015). Otros de los aspectos importantes que se recogen en el estado del arte sobre experiencias de desarrollo profesional tiene relación con la colaboración. Varios autores sostienen la importancia que ha tenido la colaboración entre colegas (Goodchild, 2011) y la colaboración entre investigadores y profesores en el contexto de formación continua (Schoenfield, 2011). Finalmente, otra dimensión que se destaca es el uso de videos en la formación de profesores (Sherin y Han, 2004; Sherin y van Es, 2009; Koh, 2015) que se asocia al trabajo vinculado al análisis en base al uso de evidencias desde contextos cercanos y reales. El uso de videos en la formación de profesores tiene un impacto directo en las concepciones de los docentes (Lipowsky, 2004).

Estos antecedentes permiten fundamentar las cuatro dimensiones que sostienen nuestro modelo de formación: conocimiento pedagógico del contenido, trabajo colaborativo, 
desarrollo profesional situado en la práctica y el trabajo basado en evidencias por medio del uso de videos. A continuación, describiremos el modelo de formación.

\section{MEJORAMIENTO DE LA EXPERIENCIA DOCENTE (MED)}

Desde el año 2010, hemos estado construyendo una metodología de formación a partir de diversos proyectos de investigación y de desarrollo, los que han implicado una formación de profesores en la temática de cada uno de estos proyectos. Entre ellos destacamos el proyecto FONIDE 511091 (2010-2011) que tuvo como foco la formación de profesores de enseñanza básica para promover el desarrollo de competencias matemáticas en el aula (Solar et al., 2011); y, recientemente, el proyecto FONDECYT de Iniciación 11130675 (20142015), que tuvo como objetivo promover en los profesores el desarrollo de la competencia de argumentación en el aula de matemáticas (Solar y Deulofeu, en prensa). En estas investigaciones, la metodología de formación MED permitió que los docentes se apropiaran de conocimiento pedagógico del contenido específico, articulado con prácticas para la gestión del aula matemática, en relación a la promoción del desarrollo de competencias matemáticas. Las experiencias descritas permitieron validar la metodología como modelo para el desarrollo profesional del profesor de matemáticas (Solar et al., 2011; Solar, Rojas, Ortiz y Ulloa, 2012; Solar y Deulofeu, en prensa).

La metodología originalmente se constituyó de 3 etapas: a) estudio de un conocimiento pedagógico del contenido (CPC) del aula de matemáticas mediante el análisis de la práctica de otros, b) análisis de la propia práctica y el diseño de clases en base al CPC en estudio, c) implementación y evaluación de clases con criterios basados en el CPC. Después de varios proyectos de aplicación, hemos visto que es mejor, desde la perspectiva de la progresión del proceso de formación, separar el análisis de la propia práctica con el diseño de clases y unir este último con la aplicación, resultando finalmente que la secuencia de formación se conforme de 4 etapas. A continuación se presenta el esquema con cada una de las 4 etapas.

Figura 1. Etapas del modelo de formación "Mejoramiento de la Experiencia Docente"

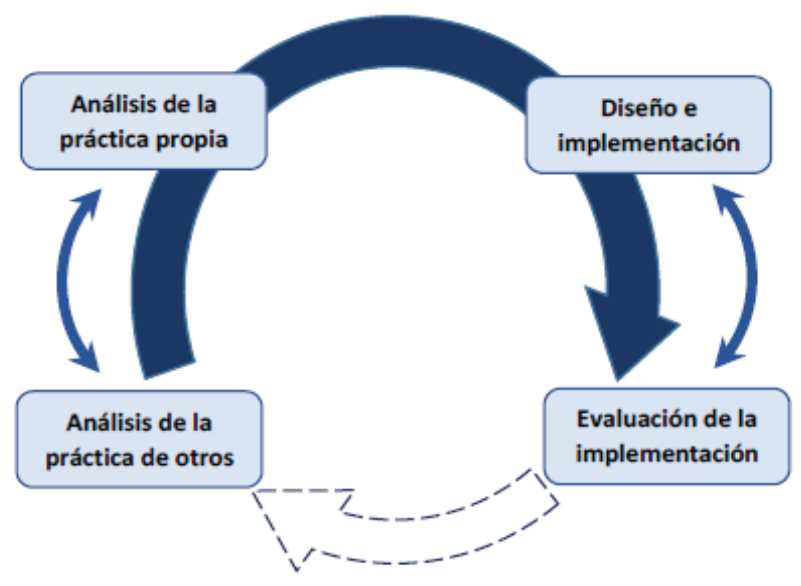


En la figura 1 se muestran las etapas de la secuencia del modelo de formación en torno a un CPC de estudio, llamado "gestión de la argumentación en el aula de matemáticas", que exponemos en este artículo, A continuación se presenta una descripción de cada una de las cuatro etapas, las que muestran cómo opera la secuencia de estudio, que busca la progresión tanto del estudio CPC, como de las tareas que los participantes emprenden durante el proceso:

- Primera etapa: Mediante el análisis de la práctica de otras clases, se plantea de qué manera se da la argumentación, acompañado de documentos que describen teóricamente la argumentación en el aula de matemáticas. En estas sesiones se ven episodios de clases en donde están presentes interacciones argumentativas y donde no están presentes dichas interacciones, y se va analizando las clases con una progresión en la complejidad en la medida que se van incorporando nuevos documentos. Una vez que se ha estudiado el tema en diferentes clases se pasa a la segunda etapa.

- Segunda etapa: Aquí el docente comienza a incorporar el CPC en estudio a sus propias prácticas de clases y además puede seguir estudiando el mismo CPC anterior o complementar con otros CPC. En nuestro caso, en esta etapa se ha pasado a estudiar las estrategias comunicativas, que si bien no se han diseñado expresamente para la argumentación, muchas de ellas son propicias para promover la argumentación en el aula de matemáticas. Varias de estas clases son observadas por el equipo de formadores para ser analizadas y ser utilizadas en el mismo proceso formación, por ello se espera que las primeras intervenciones del profesor sean vistas por ellos mismos para el estudio del CPC estrategias comunicativas.

- Tercera etapa: En ella se comienza el diseño de clases, para eso se comienza modificando planes de clases que se han visto anteriormente para que se promueva la argumentación, siguiendo con el diseño de una secuencia de clases para el CPC en estudio. En nuestro caso, los profesores diseñaron dos clases, no necesariamente secuenciadas, para promover la argumentación. Esta clases son implementadas y observadas por que el equipo de formadores para su posterior análisis.

- Cuarta etapa: Aquí se evalúa el proceso de estudio del CPC, es decir se evalúa tanto el desempeño del docente en el aula como su reflexión sobre el tema. En nuestro caso, se han evaluado las clases que se han diseñado para establecer en cuántas de estas aparece efectivamente la argumentación. La reflexión se realiza mediante un grupo focal para evaluar de qué manera los docentes reconocen la argumentación en una clase en video. Hemos identificado, además, la conveniencia de iterar estas etapas en ciclos dentro del proceso de formación, lo que supone un trabajo en espiral, en que la ejecución de cada ciclo permite remirar y profundizar en las fases anteriores.

Para comprender los criterios bajo los cuales esta metodología se organiza, es necesario también visibilizar cuatro principios orientadores del modelo de formación que 
fundamentan la propuesta y que actúan de forma transversal a la secuencia de formación. Un primer principio es que el modelo de formación es apropiado siempre y cuando exista con un Conocimiento Pedagógico del Contenido (CPC), es decir no es propicio para estudiar solo matemáticas ni tampoco para aspectos que no tengan que ver con el aprendizaje de un contenido matemático. El modelo MED se ha generado para que el profesor se apropie de conocimientos propios para enseñar la matemática, y ello se conecta muy bien con lo que Shulman ha acuñado como Conocimiento Pedagógico del Contenido. Un segundo principio del modelo de formación es el trabajo colaborativo, en que el formador va colaborando con el docente en la preparación de sus clases y va monitoreando el proceso mediante la observación de sus clases. El MED se caracteriza por problematizar situaciones de enseñanza por medio de análisis de la práctica de aula, e incentivando la reflexión permanente del profesor, teniendo como objetivo que el profesor profundice en las reflexiones que puede hacer de las prácticas tanto de sus compañeros como de otros. De esta idea se desprenden el tercer y cuarto principio, el estudio de un CPC debe hacerse de manera articulada con las experiencias de aula y el estudio de la práctica; se realiza con base a evidencias, en especial aquellas propias de su contexto.

Las etapas del MED como algunos de los cuatro principios, se pueden visualizar de alguna manera en los modelos destacados por Roesken (2011). Pero estas dimensiones no destacan las particularidades que hace que un modelo de formación tenga impacto en el profesor. Por ello, consideramos que es necesario agregar una tercera dimensión que haga mención a estas particularidades y que denominaremos como las características del modelo de formación. En este artículo describiremos cada una de las características del modelo MED por medio del proceso del estudio de la gestión de la argumentación en el aula de matemáticas.

Como contexto, cabe señalar que el estudio se realizó en el marco del proyecto FONDECYT, uno de sus objetivos siendo el de estudiar las condiciones para el desarrollo de la habilidad de argumentar en el aula de matemáticas. Para ello, se implementó un seminario de 1 año de duración, compuesto por 20 sesiones, en los que participaron 10 profesores de educación básica de la provincia de Concepción. Para el diseño del seminario se decidió utilizar el modelo de formación "Mejoramiento de Experiencia Docente" (MED) ya descrito. Se organizó un equipo de trabajo compuesto por el investigador principal y dos formadores de profesores con experiencia en formación inicial y continua.

Para analizar las características particulares del modelo de formación MED, se filmaron de forma completa todas las sesiones, en que el investigador tomaba el rol de observador no participante, mientras los formadores cumplían el rol de observadores participantes. Se analizaron las 20 sesiones con el propósito de seleccionar actividades de formación cuyos resultados permitieran representar de mejor forma los principios de la metodología, así como momentos en las que se observaran conductas y respuestas en los participantes que fuesen evidencia de reflexiones de nivel interpretativo

\section{CARACTERÍSTICAS DEL MED}

Los análisis de las sesiones del seminario permitieron identificar seis características que tienen la connotación de ser centrales para el modelo MED, las cuales son: (a) Conocimiento del grupo de profesores; (b) Estudio del conocimiento pedagógico del contenido 
(CPC); (c) Problematizar la práctica como medio para desarrollar el CPC; (d) Elicitar ideas de los profesores; (e) Implementar y evaluar propuestas de enseñanza; (f) Secuencia progresiva y articulada de temas y casos. A continuación se describen cada una de las características por medio de ejemplos en diferentes sesiones del seminario:

(a) Conocimiento del grupo de profesores: Si se pretende impactar en la formación de profesores, una primera característica es conocer las experiencias y concepciones del docente. Para ello se realiza un proceso de diagnóstico y, si es necesario, una selección de participantes. En el caso de nuestro seminario, se entrevistó a un conjunto de profesores para recabar información sobre sus concepciones acerca de la relevancia de la interacción en el aula de matemáticas. Los docentes entrevistados que no daban importancia a la socialización de las ideas de los estudiantes en el proceso de resolución de un problema no fueron seleccionados, puesto que consideramos como premisa que los profesores tenían que valorar los espacios de comunicación en el aula, ya que el periodo de 8 meses era ajustado pues ellos debían desarrollar la argumentación en sus estudiantes. Es importante señalar que esta característica de conocer al grupo de profesores no es exclusiva del inicio del proceso de formación, sino que se presenta en forma constante, ya que las distintas etapas permiten la manifestación de distintas habilidades y competencias. El proceso asociado a implementar el modelo MED requiere recopilar información constante sobre los profesores, pues esto permite diseñar sesiones de trabajo que consideren el contexto de sus participantes.

(b) Estudio del conocimiento pedagógico del contenido $(C P C)$ : Esta característica se refiere al estudio de temas asociados a conocimientos pedagógicos del contenido (CPC) que son objetivos del proceso de formación, o bien, medios para el logro de otros propósitos. Esta definición no considera por tanto el estudio de conocimientos matemáticos puros, ya que MED se ha diseñado específicamente para promover el desarrollo de conocimientos didácticos basado en la reflexión. Tampoco se incluye conocimiento pedagógico puro, por cuanto la reflexión que se propone en MED tiene una base empírica y práctica, y esta base puede ver modificada su estructura al abordar distintas disciplinas. Es evidente que el conocimiento aasociado esta propuesta es de naturaleza pedagógica del contenido, en los sentidos propuestos por Shulman (1986), Ma (2013) e Hill et al. (2008). Estos últimos, han propuesto distintas dimensiones del conocimiento matemático para la enseñanza que permiten abordar una multiplicidad de fenómenos asociados a la docencia en el aula de matemáticas. Pero las distintas implementaciones no se han limitado a los modelos señalados, sino que también se han incorporado elementos de otros modelos de desarrollo de competencias matemáticas que requieren de una gestión de clase especializada que se vincule con la estructura y naturaleza del conocimiento matemático (Rojas, Flores, Carrillo, 2015).

En nuestro seminario se estudiaron dos CPC para el desarrollo de la argumentación en el aula de matemáticas: identificación de la argumentación y estrategias comunicativas para gestionar la argumentación. Respecto al primer CPC, en las primeras sesiones se estudió la diferencia entre argumentar y explicar, así como la estructura de Toulmin para reconocer la argumentación. Un ejemplo de ello se observa en la sesión 1 (ver tabla 1), en donde se busca caracterizar y diferenciar entre explicar y argumentar. 
Tabla 1. Algunos conocimientos estudiados en el aula matemática

\begin{tabular}{|c|l|l|}
\hline Sesión & \multicolumn{1}{|c|}{ CPC estudiado } & \multicolumn{1}{c|}{ Ejemplo de actividad de formación del seminario } \\
\hline 1 & $\begin{array}{l}\text { Argumentación en el } \\
\text { aula de matemáticas }\end{array}$ & $\begin{array}{l}\text { Observe los siguientes episodios de clases: } \\
\text { Episodio 1: Niños calculando 65+65 en la pizarra. } \\
\text { Episodio 2: Niños resolviendo un problema de decrecimiento } \\
\text { exponencial. } \\
\text { Señala en cada una de los episodios si la interacción es explicativa } \\
\text { o argumentativa. }\end{array}$ \\
\hline 11 & $\begin{array}{l}\text { Estrategias comuni- } \\
\text { cativas }\end{array}$ & $\begin{array}{l}\text { Observar el Episodio 1 de la clase de Matilde. } \\
\text { En la tabla se presentan acciones docentes para cada una de las } \\
\text { cuatro estrategias seleccionadas para promover la comunicación. } \\
\text { Marque las acciones docentes que se observan de manera } \\
\text { destacada en el episodio transcrito de la clase observada. Marque } \\
\text { algunas evidencias en el documento para justificar la presencia de } \\
\text { las acciones docentes señaladas. }\end{array}$ \\
\hline 13 & $\begin{array}{l}\text { Rol de las tareas ma- } \\
\text { temáticas en el desa- } \\
\text { rrollo de la argumen- } \\
\text { tación. }\end{array}$ & $\begin{array}{l}\text { 1. Lea el documento (planificación), y señale cuáles son los } \\
\text { elementos que promueven o limitan los procesos argumentativos. } \\
\text { 2. Observe el episodio de clases. Analice la gestión de la } \\
\text { profesora, respecto de lo planificado. ¿Se observan efectos sobre } \\
\text { el proceso argumentativo? ¿Cuáles? }\end{array}$ \\
\hline
\end{tabular}

En la sesión 1 episodio 1, los dos niños que aparecen en el episodio están discutiendo sobre la validez de un procedimiento para calcular $65+65$, en que uno de ellos está refutando el procedimiento incorrecto utilizado por el otro niño. Por lo tanto, se presenta una interacción argumentativa. En cambio, en el episodio 2, una estudiante de $8^{\circ}$ básico describe en la pizarra al resto del curso su procedimiento para resolver un problema de decrecimiento exponencial. Si bien la descripción incorporaideas matemáticas correctamente fundamentadas, la intervención de la estudiante corresponde a una explicación porque no hay una discusión de las ideas y no pretende convencer al curso defendiendo su posición sino que describe sus ideas. Muchos profesores tienen la concepción que una explicación con ideas fundamentadas es una argumentación y cuando comparan con un episodio en que hay contraposición de ideas cuestionan esta idea de argumentación. Sobre la mitad del proceso de formación se inició el estudio al segundo CPC: estrategias comunicativas para gestionar la argumentación en el aula de matemáticas. En la tabla 1 se muestran las instrucciones de la sesión 11, en que una vez visto el episodio de clase de una de las profesoras que participan en el seminario, se les solicita que señalen cuáles son las acciones docentes asociadas a las estrategias comunicativas observadas en el episodio. Cabe señalar que en el episodio existe argumentación, y se espera que los profesores reconozcan ciertas acciones docentes asociadas a las estrategias comunicativas de: promover la participación, gestionar el error, y preguntas clave. En la sesión 13 aparece el rol de las tareas matemáticas para el desarrollo de la argumentación. A partir de una análisis de una planificación, el propósito de la sesión es que los docentes reconozcan que las características de las tareas matemáticas pueden limitar o promover la argumentación en la clase, tareas que se pueden resolver mediante 
diferentes procedimientos, o de respuesta abierta, o que invita a diferentes posturas, son características de tareas que promueven la argumentación en el aula de matemáticas.

(c) Problematizar la práctica como medio para desarrollar el CPC: Estudiar con los profesores el conocimiento especializado para enseñar matemática, tal como se describió previamente, se realiza en contextos de estudio de casos, lo que permite el análisis de la práctica propia y las de otros. Más aun, el ciclo de implementación de la metodología avanza hacia la preparación e implementación de clases, lo que transforma el análisis de la práctica de otros en una planificación y reflexión de la práctica propia. Se observa, entonces, un conjunto de tareas basadas en acciones que el profesor efectivamente realiza en su práctica, tareas que constituyen una problemática que requiere de acciones y contextos progresivos que van incrementando la complejidad de análisis. Esto se evidencia en el uso de las cuatro etapas en el seminario: en las sesiones 1 a las 3 se analizó la práctica de otros, entre las sesiones 4 a las 14 se analizó la práctica propia. Entre las sesiones 15 a la 19 se planificó la práctica propia y en la sesión 20 se evaluó la práctica propia. En la tabla 2 se puede evidenciar que en la sesión 1 se utilizaron episodios de clase de otros profesores, mientras que en la sesión 11 se utilizó un episodio de clase de una de las profesoras que se grabó anteriormente.

Para ofrecer mayor claridad sobre la forma en que esta característica opera, desarrollaremos lo ocurrido en una de las sesiones del seminario, en donde las profesoras fueron problematizadas respecto del diseño de actividades, en donde analizaron, evaluaron y modificaron el conjunto de decisiones asociadas a dicha acción. Las participantes habían estudiado el modelo de argumentación, distintas estrategias comunicativas para promover argumentación y ciertas características de las consignas que generan oportunidades para la discusión. En un contexto tradicional de formación, en donde se simplifica el fenómeno de preparación y gestión de la enseñanza, operaría el supuesto de que las profesoras ya están preparadas para implementar exitosamente clases con propósitos de desarrollo de la competencia argumentación. Sabemos que no es así, y la sesión 9 opera para problematizar la articulación de los elementos estudiados en las sesiones anteriores. Se les solicitó a las profesoras que diseñaran o seleccionaran una actividad nuclear que buscara promover la argumentación, definiendo la consigna de la tarea, su gestión, y las estrategias a emprender, describiéndolas en un formato de plan de clases. Una vez finalizadas, las profesoras tuvieron que co-evaluar las producciones, utilizando una pauta que buscaba identificar si los planes de clase anticipaban distintos argumentos y sus elementos (conclusiones, explicaciones, refutadores, etc.), y si las estrategias se habían seleccionado utilizando criterios generales, o usando criterios pedagógicos del contenido. Se observó que las planificaciones usaban criterios generales; por ejemplo, una profesora propuso como actividad "Determinar la fracción que se debe quitar a la suma $3 / 9+2 / 3$ para que el resultado sea 1/9", describiendo como gestión de clase que los alumnos "planteen diferentes estrategias, utilicen los términos matemáticos pertinentes, y expongan estrategias". El análisis entre pares identificó que no se habían anticipado las posibles respuestas, que no se habían propuesto errores plausibles ni distintas estrategias correctas, y que las estrategias de gestión eran independientes de la naturaleza de las respuestas, identificando por ejemplo que una exposición final de las estrategias no promovería argumentación en los estudiantes. La toma de decisiones y el reconocimiento de que ciertas prácticas tradicionales de enseñanza requieren ser modificadas fue crucial dentro del proceso de formación de las profesoras. 
La problematización de la práctica busca recoger el estudio de problemas matemáticos en el contexto mismo de la enseñanza, y en ausencia de respuestas "correctas", permite reflexionar sobre criterios que se adaptan a las distintas realidades de las participantes.

(d) Elicitar ideas de los profesores: se pudo observar que los profesores participantes logran dar a conocer en profundidad el conocimiento especializado para enseñar matemática, para posteriormente poner en acción sus ideas. Lo anterior se logra a través de dos mecanismos: iniciar siempre con una problematización, y la gestión del formador. El primer mecanismo por su carácter desafiante, promueve la comunicación y discusión de sus ideas, viéndose los profesores en la necesidad de argumentar y precisar sus afirmaciones respecto de sus creencias. Esta problematización se plantea en un contexto específico y real, en donde las tareas y preguntas son formuladas desde una perspectiva de tomar posición respecto a algún elemento. Esto promueve la manifestación de al menos dos elementos que en nuestra opinión son muy relevantes: (1) en primer lugar, se observan manifestaciones de las creencias de los profesores, en vez de emitir respuestas que el docente supone que los formadores esperan; (2) en segundo lugar, permite asignar tareas que van más allá del análisis de la gestión de otros.

En la tabla 2 se observan algunos ejemplos de actividades de problematización que responden a estos elementos. Se observa que las preguntas orientadoras se asocian a problemáticas específicas en contextos reales e inducen la toma de decisiones y a la reflexión, permitiendo la vinculación reflexiva con la práctica propia, con lo que la elicitación de las ideas de los participantes se vuelve natural. Cabe consignar que, en las primeras etapas, los elementos recién mencionados no necesariamente se dan en forma espontánea, por lo que la característica requiere del segundo mecanismo: la acción del formador que está a cargo de la implementación de la metodología en las sesiones.

Tabla 2. Ejemplos de primer mecanismo: "Iniciar siempre con una situación problemática"

\begin{tabular}{|c|l|}
\hline Sesión & \multicolumn{1}{c|}{ Pregunta orientadora del problema } \\
\hline 10 & $\begin{array}{l}\text { ¿Qué elementos de la gestión del profesor observado modificaría usted para promover la } \\
\text { manifestación de interacciones en niños que presenten la estructura argumentativa } \\
\text { completa? ¿Por qué? }\end{array}$ \\
\hline 13 & $\begin{array}{l}\text { Luego de estudiar la planificación de clase, y de observar clase, ¿está de acuerdo con las } \\
\text { modificaciones que la profesora realizó respecto de lo planeado? ¿Qué elementos habría } \\
\text { mantenido usted, y por qué? }\end{array}$ \\
\hline
\end{tabular}

Este segundo mecanismo, la gestión del formador, por su carácter inductivo, promueve la comunicación, verificación y reflexión de sus ideas, viéndose en la necesidad de contrastar y modificar sus afirmaciones respecto del marco teórico. El formador no es solo un mediador, sino que cuestiona y profundiza en las ideas de los profesores, verificando si las interpretaciones son adecuadas, generando debates, buscando comprensión común del grupo para ciertas ideas, ya sean presentadas a priori, o emergentes. Así, es el formador quien busca la reflexión de los participantes sobre tareas que son propias del 
diseño, implementación y evaluación de secuencias didácticas. A continuación se presenta un extracto de la sesión 13, en donde se observa este mecanismo, en un diálogo entre Bernardita y el formador:
Formador:
¿Qué aspecto de la actividad promueve argumentación?
Bernardita:
El que se pida que expliquen cómo lo están haciendo.
Formador: ¿Se observa en el video que tu gestión provoca esto?
Bernardita: Yo creo que no porque... No está como directo... Yo vi a un chico resolver, pero no pregunté más generalizado, “¿cómo lo hiciste tú?”, como para haber visto más opiniones de los chicos antes de resolver el ejercicio en sí. No aparece ahí que haya habido más opiniones respecto de lo que es la estimación.
Formador: Pero en esta clase ¿revisaste lo que los alumnos tenían en sus cuadernos?
Bernardita: Si, de hecho... Pero... Pienso que debería haber mandado a un chico a la pizarra que la haya hecho equivocado.
Formador: Pero la niña que enviaste no lo hizo equivocado
Bernardita: No, pero primero, debí enviar alguien antes que la niña. Entonces... yo lo hice al revés. Envié a la niña después con el error, pero, a lo mejor, para que hubiera habido más discusión... debí enviar el error primero. Entonces ahí habría podido pedirles a más chicos para que hubiera un debate entre ellos.

El proceso de elicitación de ideas de profesores se planifica en la metodología. Las acciones de diseño y gestión de las sesiones se articulan entre sí. La experiencia del equipo a cargo de estas acciones pasa a ser relevante para la selección de las tareas que los profesores deberán realizar, así como de la elección de los episodios de clases que serán objeto del estudio de caso.

(e) Implementar y evaluar propuestas de enseñanza: Un elemento central del modelo MED es la implementación y evaluación de propuestas propias. Esta implementación busca poner en juego el conocimiento que se ha estado estudiando y las propuestas pueden ser de distinta envergadura, dependiendo de la naturaleza del proceso específico de formación continua. El abanico puede ir desde la preparación de la gestión de una actividad hasta la planificación de una unidad didáctica. En el caso del seminario, luego de analizar la estructura de la argumentación, el análisis de clases de niños argumentando, evaluando planificaciones y gestión de otros, los docentes planifican una clase que será realizada con sus estudiantes. La lección es planeada en un formato que permita poner énfasis en los elementos de estudio del seminario. En nuestro caso, se pedía incluir, a continuación de la actividad central para promover argumentación en el aula, el conjunto de respuestas y posiciones esperadas que podrían surgir los estudiantes. Aquí no se pedía el universo completo de respuestas posibles, sino un set de afirmaciones plausibles de ser propuesta por el grupo de alumnos del profesor. A continuación, se solicitaba describir acciones docentes específicas para promover la contraposición de ideas, como por ejemplo, modificar las condiciones del problema, registrar respuestas seleccionadas en la pizarra antes de su socialización, simular ignorancia o validar momentáneamente la respuesta incorrecta frente a un problema, entre otras. La elección de estas estrategias de gestión tiene un componente empírico, pues se le pide a los docentes que anticipen el escenario a partir del conocimiento que tiene del grupo específico de niños. 
El plan de clase es retroalimentado por el equipo de formadores, para luego ser implementada. Esta clase se filma y analiza, identificando los aciertos y problemáticas asociadas a la implementación del plan. Estos elementos son utilizados para la edición de un clip que permita, en sesiones posteriores, analizar y evaluar la implementación por parte del grupo completo de profesores.

A modo de ejemplo, ilustramos con el caso de Mónica, profesora de $6^{\circ}$ básico, quien diseñó una clase de clasificación de cuadriláteros. Ella planeó la clase para, entre otras cosas, generar una discusión respecto a si un cuadrilátero que tiene forma de rombo puede ser un cuadrado al igual que otro cuadrilátero que sí parece cuadrado ya que está ubicado en su posición estándar. Si bien se produjo la discusión planificada, hubo una respuesta que no esperaba Mónica, un estudiante dice que cuando el rombo gira, se transforma en cuadrado y el que era un cuadrado se transforma en rombo. Esta intervención del estudiante, desencadena en Mónica modificar la gestión de la clase, incorporando la nueva respuesta en la discusión para llegar a la conclusión que una figura no cambia al girar. El análisis de esta clase en el seminario, por parte del grupo de profesores, permitió evidenciar que la respuesta inesperada del estudiante incentivó a los niños a participar del debate, promoviendo en ellos proveer argumentos más precisos a favor o en contra, lo que fue valorado muy positivamente por todos los profesores del seminario. La contraposición de opiniones permitió a Mónica valorar desde otra perspectiva el resultado de su gestión, mientras que al resto del grupo permitió comprender la lógica y propósitos detrás de las decisiones de Mónica, teniendo la oportunidad de preguntar de inmediato si esperaba o no ciertas reacciones.

(f) Secuencia progresiva y articulada de temas y casos: Esta característica acompaña la evolución estructural de las distintas etapas del modelo MED. El diseño de las sesiones busca que los profesores vivan un continuo de formación, lo que requiere que las sesiones dentro de cada etapa progresen, tanto en la complejidad del conocimiento como del análisis. Lo anterior se puede observar en la tabla 3 donde se muestra la secuencia de tareas asociadas al estudio de casos que permite conectar las etapas 2 y 3 de esta implementación particular del MED.

Tabla 3. Muestra de secuencia de tareas de estudio de casos de sesiones consecutivas

\begin{tabular}{|l|l|l|}
\hline \multirow{2}{*}{ ETAPA 2 } & S12 & $\begin{array}{l}\text { Análisis de la gestión de las condiciones didácticas de una actividad, que } \\
\text { promueven argumentación. }\end{array}$ \\
\cline { 2 - 3 } & S13 & $\begin{array}{l}\text { Análisis de la relación entre las condiciones didácticas de una actividad, y la } \\
\text { gestión de aula y acciones docentes que promueven argumentación. }\end{array}$ \\
\cline { 2 - 3 } & S14 & $\begin{array}{l}\text { Frente a una actividad con condiciones didácticas adecuadas, análisis de las } \\
\text { características de la gestión especializada de clase para efectivamente promover } \\
\text { procesos argumentativos. }\end{array}$ \\
\hline \multirow{2}{*}{ ETAPA 3 } & S15 & $\begin{array}{l}\text { Frente a una actividad con condiciones didácticas adecuadas, diseñar la gestión } \\
\text { especializada de clase para promover efectivamente procesos argumentativos. }\end{array}$ \\
\cline { 2 - 3 } & S16 & $\begin{array}{l}\text { Diseñar una actividad, con condiciones didácticas específicas, y su gestión } \\
\text { especializada de clase para promover efectivamente procesos argumentativos. }\end{array}$ \\
\hline
\end{tabular}




\section{DISCUSIÓN Y CONCLUSIONES}

En el análisis, nos hemos centrado en las características del modelo de formación por medio de evidencias de cada una de estas características en sesiones de un proceso de formación. Ahora bien, ¿cuál es la ventaja del modelo MED en comparación a otros que hemos descrito? ¿cuáles han sido los resultados entre los profesores y sus prácticas? ¿con que temas se puede usar el MED? ¿quién lo puede usar? En torno a estas cuatro preguntas desarrollaremos la discusión de los resultados.

\subsection{MED EN RELACIÓN OTROS MODELOS DE FORMACIÓN CONTINUA DE PROFESORES}

Los procesos de formación de profesores de matemáticas, que hemos desarrollado desde el 2010 a través de MED, han logrado superar los enfoques tradicionales de formación, los cuales tienen la concepción central de que primero se deben modificar los conocimientos y creencias de los profesores, y que ello tiene como consecuencia lineal una modificación de sus prácticas. En cambio, en el MED, el estudio del CPC y el análisis de la práctica se articulan progresivamente, en el entendido que esta relación continua favorece y nutre los cambios y reflexiones sobre ambos elementos. Esta reflexión y experimentación permite en primer lugar hacer consciente al docente de las decisiones e intenciones detrás de estas, de modo de poder evaluarlas de forma más transparente. Por ello, compartimos con Clarke y Hollinsworth (2002) que un modelo de formación debe ser interconectado entre el trabajo de la práctica con la reflexión. En nuestro modelo, la práctica temprana que tienen los docentes en la segunda etapa del MED, y sobre todo la práctica planificada en la tercera etapa, tiene un fenómeno ya señalado por Guskey (2000): los profesores, al analizar sus propias clases en el seminario, recogen evidencias de cambio en la propia práctica y en los estudiantes, impactando en las concepciones de estos docentes y en consecuencia en la apropiación del CPC en juego.

Por otra parte, el MED ha validado el uso de videos y de estudio de casos como una fuente importante de reflexión sobre la práctica. Más aun, la secuencia de un análisis de la práctica de otros, que avanza hacia el análisis de la práctica de un colega y, eventualmente la propia, va dotando de objetividad al proceso, al refinar los criterios de observación y evaluación, lo que es consistente con otros resultados de investigación (Sherin y Han, 2004; Sherin y van Es, 2009; van Es y Sherin, 2010), pero este hecho solo se debe entender considerando que tanto el diseño de las actividades de formación, como la gestión de las sesiones por parte de los formadores, articulan consistentemente un Conocimiento Pedagógico del Contenido con un análisis reflexivo de la práctica. Asimismo, compartimos con Schoenfeld (2011) el trabajar con los docentes la toma de decisiones. Consideramos que el foco de orientaciones que plantea Schoenfeld en su modelo de formación continua es clave en los procesos de cambio del profesor, pues supera el hecho de focalizarse solo en las concepciones pues también considera la toma de decisiones del docente. En el MED, la característica de problematizar la práctica como medio para desarrollar el CPC promueve que el docente tome decisiones para que se produzca un conflicto cognitivo en el profesor, lo que favorece consciencia de su aprendizaje respecto al CPC en juego.

Lipowsky (2004) sostiene que en un proceso de formación continua, el uso de videos afecta directamente las creencias de los profesores. En base a nuestra experiencia, hemos evidenciado que además el uso de los videos es una manera de invitar al profesor a que 
realice ese tipo de práctica. Por eso es importante en el modelo MED que el profesor primero vea la práctica de otros, para que luego se focalice en su propia práctica. En consecuencia, el uso de videos no solo impacta en las concepciones de los profesores, sino también a su propia práctica.

Los modelos de formación que hemos analizado, por lo general, tienen etapas y principios que los conforman, pero no es usual encontrar un conjunto de características que describan en detalle cómo funciona el modelo en un proceso de formación. En el caso del MED, de las seis características que hemos descrito, consideramos que la práctica como medio para desarrollar el CPC, elicitar las ideas de los profesores, y una secuencia progresiva y articulada de temas y casos, son características diferenciadores con otros modelos de formación continua, en donde no promueven que el profesor exponga sus ideas como parte del proceso de apropiación de un CPC, más bien se espera que reaccionen ante un CPC dado. Es común encontrarse con experiencias de asesoramiento en que el formador observa la clase de un profesor y retroalimenta al docente en base a una pauta de evaluación con criterios preestablecidos, indicando los elementos observados y no observados en la clase. Consideramos que este tipo de retroalimentación no produce cambios en los profesores. En nuestro modelo MED, la retroalimentación se hace en el seminario a través del análisis de un episodio de clase en que mediante la problematización y la gestión del formador, se promueve que el profesor reconozca los elementos de su práctica, elicitando así sus ideas (opiniones, decisiones, etc.) y luego contrastándolas con la evidencia. El hecho que ese análisis sea grupal permite que por lo general la evaluación de la clase realizada previamente por el formador, sea similar a la obtenida por el grupo de profesores en el seminario. Con ello hemos evidenciado que el docente realmente se apropia de las acciones que dieron resultado y toma conciencia de las acciones que no contribuyen a gestionar la clase según el propósito establecido.

\subsection{EFICACIA DEL MODELO MED}

Las experiencias de formación, que por lo general se han desarrollado, no han fracasado por un cuestionamiento a su estructura tradicional -focalizarse en el conocimiento y concepciones, y luego en la práctica-, sino que en el escaso impacto en cambiar las prácticas de los docentes y en no mejorar los desempeños de los estudiantes (Roesken, 2011). En las experiencias de formación continua de profesores en que hemos implementado MED, hemos constatado cambios en las prácticas y concepciones de los profesores, mostrando así una apropiación del CPC. En Solar y Deulofeu (en prensa) se muestra de qué manera los profesores en un inicio del seminario tienen dificultades tanto en no reconocer la argumentación como en gestionar de manera argumentativa la clases de matemáticas. En cambio, ya transcurridos unos 8 meses de participación en el seminario, prácticamente todos los docentes identifican la argumentación en clases y son capaces de desarrollar argumentación en el aula de matemáticas. Ello nos ha permitido sostener que MED es un modelo experimentalmente validado para producir cambios en los profesores, y ello es un gran avance respecto a las experiencias de formación continua que por lo general les es muy difícil tener evidencia de cambios concretos en el profesor. El próximo paso es obtener resultados sobre los desempeños de los estudiantes, para ello es necesario que se cumplan condiciones que hasta ahora no hemos tenido en los proyectos desarrollados: asesoría a las escuelas y monitoreo en los profesores y estudiantes por un plazo mínimo de dos años. El 
grupo de investigación COMMAT tiene como objetivo futuro esta problemática de determinar el impacto de MED en los estudiantes y para ello ya se están preparando proyectos investigación en esta línea.

\subsection{DESARROLLO DEL CPC}

Las experiencias de formación continua que hemos desarrollado por medio del MED han mostrado que los docentes se apropian del Conocimiento Pedagógico del Contenido (CPC): desarrollo de las competencias matemáticas, la incorporación del álgebra temprana (Early algebra) en los primeros niveles del básica, la argumentación en el aula de matemática, entre otros. En el primer proyecto, del que emergió el MED, obtuvimos como conclusión que los cambios en el profesor no solo requieren de un CPC acorde a las necesidades del docente sino una metodología para que el docente se apropie de dicho CPC (Solar et al., 2012). Actualmente, consideramos que el valor del modelo de formación es crucial en los proceso de cambio. Es habitual pensar que la efectividad de un modelo de formación de profesores pasa más por los CPC en juego que por la forma de trabajar con los profesores. Nosotros consideramos que los temas pueden variar en función de las necesidades del profesor o sistema. Más aún, el modelo MED puede ser aplicado después de un diagnóstico al establecimiento educacional y, a partir de los resultados, decidir cuáles pueden ser los temas de formación pertinente. En esta misma lógica, incluso no sería necesario tener un CPC predominante en el proceso de formación, sino que pueden ser temas puntuales como, por ejemplo, la gestión del error o la elicitación de ideas de los estudiantes, en que se pueden utilizar videos de otros profesores en que se vean estas prácticas para que luego los mismos docentes se aproximen a dichas gestiones de clases con intervenciones puntuales donde discutan sus experiencias para luego planificar y evaluar.

Por otra parte, una limitación del modelo MED es que opera solo bajo conocimientos de tipo CPC pues el modelo no está diseñado para promover apropiación de conocimiento matemático puro o formal, ni desarrollo de razonamiento matemático, entendido como el proceso de aplicación de la lógica deductiva de la disciplina. Esta limitación se explica por la dificultad de articular el conocimiento matemático formal con las prácticas de enseñanza, sin contar con un conocimiento que medie entre ambos, rol que cumple el CPC.

\subsection{TRANSFERIBILIDAD DE MED}

Dado que el modelo de formación Mejoramiento de la Experiencia Docente (MED) ha sido experimentado por medio de diferentes CPC, consideramos que este es transferible a instituciones que realicen Asistencia Técnica Educativa a establecimientos educacionales. Durante el año 2015, hemos llevado a cabo una experiencia de transferibilidad del modelo de formación MED mediante un proyecto piloto con una ATE en que sus asesores utilizaron MED para asesorar a los profesores de establecimiento educacional en matemáticas.

Los resultados indican que los asesores de la ATE percibieron el impacto del modelo de formación MED en los profesores de matemáticas y los propios docentes tuvieron una actitud positiva sobre los temas de formación: argumentación en el aula de matemáticas y gestión del error, logrando que estuvieran dispuestos a grabarse para compartir sus clases entre sus colegas. Esta percepción fue muy valorada por los relatores de la ATE que en experiencias anteriores habían visto como los docentes eran resistentes a ser grabados y 
en aplicar las estrategias promovidas por la ATE. Durante el 2016 se sigue escalando la transferibilidad del modelo MED, con otras ATEs que así lo requieran. Con ello, esperamos contribuir a los procesos de certificación que está liderando el Mineduc a la calidad en las asesorías que ofrecen las ATE.

\section{REFERENCIAS BIBLIOGRÁFICAS}

Agencia de Calidad de la Educación (2013). Informe Nacional de Resultados SIMCE 2012. Santiago: Ministerio de Educación de Chile. Recuperado el 05 de febrero de 2016 desde https://s3.amazonaws.com/archivos.agenciaeducacion.cl/documentos-web/Informe_Tecnico_ Simce_2012.pdf

An, S. y Peter-Koop. A. (2015). In-Service Education, Professional Development of Mathematics Teachers. In S. Cho (Ed). The Proceedings of the 12th International Congress on Mathematical Education. Intellectual and Attitudinal Challenges (pp. 513-516). Heidelberg New York Dordrecht London: Springer Open.

Alvarado, M., Cabezas, G., Falck, D. y Ortega, M. E. (2012). La Evaluación Docente y sus instrumentos: Discriminación del desempeño docente y asociación con los resultados de los estudiantes. Santiago: Ministerio de Educación de Chile.

Ávalos, B. y Matus, C. (2010). La Formación Inicial Docente en Chile desde una Perspectiva Internacional. (Informe Nacional del Estudio Internacional IEA TEDS M). Santiago: Ministerio de Educación de Chile.

Bellei, C., Osses, A. y Valenzuela, J. P. (2010). Asistencia Técnica Educativa: de la Intuición a la Evidencia. Santiago: Centro de Investigación Avanzada en Educación de la Universidad de Chile y Ocholibros Editores.

Bellei, C., Raczynski, D. y Osses, A. (2010). ¿Qué hemos aprendido sobre programas de asistencia técnica educativa? En C. Bellei (Ed), Asistencia Educativa en Chile: ¿Aporte al mejoramiento Escolar? Santiago: Editorial Ocholibros.

Bellei, C., Contreras, D. y Valenzuela, J.P. (2008). Debate sobre la educación chilena y propuestas para el cambio. En C. Bellei, C. D. Contreras y J.P Valenzuela (Eds.) La Agenda Pendiente en Educación. Profesores, administradores y recursos: propuestas para la nueva arquitectura de la educación chilena. (pp. 13-47) Santiago: Universidad de Chile y Ocholibros Editores.

Cho, S. J. (2015). The Proceedings of the 12th International Congress on Mathematical Education. Intellectual and Attitudinal Challenges. Heidelberg New York Dordrecht London: Springer Open.

Clarke, D., Hollinsworth, H. (2002). Elaborating a model of teacher professional growth. Teacher and Teacher Education, 18, 947-967.

Donoso, S. (2008). Políticas de perfeccionamiento de los docentes en Chile 1990 - 2005: silencios y proyecciones. En C. Bellei, C. D. Contreras y J.P Valenzuela (Eds.) La Agenda Pendiente en Educación. Profesores, administradores y recursos: propuestas para la nueva arquitectura de la educación chilena. (pp. 79-103). Santiago: Unicef.

Goodchild, S. (2014). Mathematics teaching development: learning from developmental research in Norway. ZDM Mathematics Education, 46:305-316.

Guskey, T. R. (2000). Evaluating professional development. California: Corwin Press.

Hill, H., Ball, D.L. y Schilling, S. (2008). Unpacking pedagogical content knowledge: Conceptualizing and measuring teachers' topic-specific knowledge of students. Journal for Research in Mathematics Education, 39(4), 372-400.

Koh, K. (2015). The Use of Video Technology in Pre-service Teacher Education and In-service Teacher Professional Development. In S. Fong. Cases of Mathematics Professional Development 
in East Asian Countries Using Video to Support Grounded Analysis. (pp. 229-248). Heidelberg New York Dordrecht London: Springer Open.

Krainer, K. (2002). Investigation into practice as a powerful means of promoting (student) teachers' professional growth. In J. Novotná (Ed.), European research in mathematics education II. Proceedings of the second conference of the European Society for Research in Mathematics Education (pp. 281-291). Prague: Charles University.

Lave, J. y Wenger, E. (1991). Situated Learning: Legitimate Peripheral Participation. Cambridge: Cambridge University Press.

Lipowsky, F. (2004). Was macht Fortbildung fuer Lehrkraefte erfolgreich? Befunde der Forschung und moegliche Konsequenzen fuer die Praxis. Die Deutsche Schule, 96, 462-479.

Llinares, S., y Krainer, K. (2006). Professional aspects of teaching mathematics. In A. Gutiérrez y P. Boero (Eds.), Handbook of research on the psychology of Mathematics Education. Past, present and future (pp. 429-459). Rotterdam: Sense Publishers.

Ma, L. (2013). Conocimiento profundo de las Matemáticas Fundamentales. Santiago: Academia Chilena de Ciencias.

Ministerio de Educación de Chile (2005). Informe Comisión sobre Formación Inicial Docente. Serie Bicentenario. Santiago: Ministerio de Educación de Chile.

OECD (2013). PISA 2012 Assessment and Analytical Framework: Mathematics, Reading, Science, Problem Solving and Financial Literacy. OECD Publishing. Recuperado el 05 de febrero de 2016 desde http://dx.doi.org/10.1787/9789264190511-en

Roesken, B. (2011). Hidden Dimensions in the Professional Development of Mathematics Teachers. In-service Education for and with teachers. Rotterdam: Sense Publishers.

Rojas, N., Flores, P., y Carrillo, J. (2015). Conocimiento especializado de un profesor de matemáticas de educación primaria al enseñar los números racionales. Bolema, 29 (51),143-167.

Sherin, M. G. y Han, S. Y. (2004). Teacher learning in the context of a video club. Teaching and Teacher Education, 20, 163-183.

Sherin, M. G. y van Es, E. A. (2009). Effects of video club participation on teachers' professional vision. Journal of Teacher Education, 60(1), 20-37.

Schoenfeld, A. (2011). Toward professional development for teachers grounded in a theory of decision making. ZDM Mathematics Education, 43, 457-469.

Shulman, L. (1986). Those Who Understand: Knowledge growth in Teaching. Educational Researcher, 15(2), 4-14.

Solar, H., Espinoza, L., Rojas, F., Ortiz, A., González, E. y Ulloa, R. (2011). Propuesta metodológica de trabajo docente para promover competencias matemáticas en el aula, basadas en un Modelo de Competencia Matemática (MCM). Informe final Proyecto Fonide 511091. Santiago: Ministerio de Educación de Chile.

Solar, H., Rojas, F., Ortiz, A. y Ulloa, R. (2012). Reflexión docente y competencias matemáticas: un modelo de trabajo con docentes. RECHIEM: Revista Chilena de Educación Matemática, 6(1), 257-267.

Solar, H. y Deulofeu, J. (en prensa). Condiciones para promover el desarrollo de la competencia de argumentación en el aula de matemáticas. Bolema.

Van Es, E. A. y Sherin, M. G. (2010). The influence of video clubs on teachers' thinking and practice. Journal of Mathematics Teacher Education, 13(2), 155-176.

Valenzuela, J.P., Villarroel, G. y Villalobos, C. (2013). Ley de Subvención Escolar Preferencial (SEP): algunos resultados preliminares de su implementación. Pensamiento Educativo: Revista de Investigación Educacional Latinoamericana, 50(2), 113-131.

Vergara, C. y Cofré, H. (2014). Conocimiento pedagógico del contenido: ¿el paradigma perdido en la formación inicial y continua de profesores en chile? Revista Estudios Pedagógicos, 15 (Número Especial 1), 323-338. 